The Astrophysical Journal Supplement Series, 246:12 (14Pp), 2020 January

Preprint typeset using LATEX style emulateapj v. 01/23/15

\title{
COMPACT GROUPS OF GALAXIES IN SLOAN DIGITAL SKY SURVEY AND LAMOST SPECTRAL SURVEY: I. THE CATALOGUES
}

\author{
Yun-LiAng Zheng (郑云亮 $)^{1,2}$, SHI-Yin SHEN (沈世银) ${ }^{1,3}$ \\ (Received -; Accepted 2019-11-23) \\ The Astrophysical Journal Supplement Series, 246:12 (14pp), 2020 January
}

\begin{abstract}
A compact group (CG) is a kind of special galaxy system where the galaxy members are separated at the distances of the order of galaxy size. The strong interaction between the galaxy members makes CGs ideal labs for studying the environmental effects on galaxy evolution. The traditional photometric selection algorithm biases against the CG candidates at low redshifts, while the spectroscopic identification technique is affected by the spectroscopic incompleteness of sample galaxies and typically biases against the high redshift candidates. In this study, we combine these two methods and select CGs in the main galaxy sample of the Sloan Digital Sky Survey, where we also have taken the advantages of the complementary redshift measurements from the LAMOST spectral and GAMA surveys. We have obtained the largest and most complete CG samples to date. Our samples include 6,144 CGs and 8,022 CG candidates, which are unique in the studies of the nature of the CGs and the evolution of the galaxies inside.
\end{abstract}

Subject headings: catalogs - galaxies: groups: general - surveys

\section{INTRODUCTION}

Galaxies are the building blocks of the visible universe, inhabiting a variety of environments from fields to galaxy clusters. Observations show that over half of the galaxies are located in group systems which have members from a few to dozens (Huchra \& Geller 1982,Yang et al. 2007,Tempel et al. 2012). A compact Group of galaxies (hereafter CG) is an extreme case of groups which contains a few member galaxies separated by projected distance of the order of galaxy size. CG is believed to represent an environment where tidal interaction (Coziol \& Plauchu-Frayn 2007), harassment (Moore et al. 1998) and galaxy merging (Barnes 1989) are much more active than in a normal/loose group of galaxies. The high density and low velocity dispersion make CG to be an ideal laboratory for studying galaxy interaction and the merging process. Indeed, both simulations (Brasseur et al. 2009) and observations (Lee et al. 2004, Deng et al. 2008) have shown that the fraction of early-type galaxies in CGs is significantly higher than the counterparts in normal groups and fields. Coenda et al. (2012) found that galaxies in CGs have systematically larger concentration index and higher surface brightness and further concluded that the star-forming galaxies are more likely to be quenched in the CG environment.

In the aspect of theoretical modeling, we are not very clear about the formation channel of CGs. Given the short merging timescale of galaxies in the CG environment, the occurrence of CGs are tightly correlated with their formation process and effective lifetime. Diaferio et al. (1994) proposed that CGs are embedded in larger systems and so that they can be constantly replenished

\footnotetext{
${ }^{1}$ Key Laboratory for Research in Galaxies and Cosmology, Shanghai Astronomical Observatory, Chinese Academy of Sciences, 80 Nandan Road, Shanghai, China, 200030, ssy@shao.ac.cn

${ }^{2}$ University of the Chinese Academy of Science, No.19A Yuquan Road, Beijing, China, 100049

${ }^{3}$ Key Lab for Astrophysics, Shanghai, China, 200034
}

from neighboring galaxies. Sohn et al. (2016) found that the occurrence of CGs has no significant change with redshift and thus suggest a long life span of CGs. Also, CGs were once believed to be the progenitors of fossil groups (Ponman et al. 1994), but later Farhang et al. (2017) suggested that although CGs are systematically younger than fossil groups, their evolutionary paths are different and the evolution of CGs will not lead to the formation of the fossil groups.

To further shed light on the formation and evolution path of the CGs, a large sample of CGs with redshift measurements and well-defined selection effects are required, which is not a easy task even in these days with many modern galaxy surveys. Historically, CG samples were constructed based on photometric surveys. Hickson (1982) first introduced a set of criteria based on photometric information and identified 100 CGs (HCGs) from the Palomar Observatory Sky Survey. The three Hickson's criteria are:

1. Richness: $N(\Delta m \leq 3) \geq 4$

2. Isolation: $\theta_{n}(\Delta m \leq 3) \geq 3 \theta_{G}$

3. Compactness: $\mu \leq 26.0 \mathrm{mag} \operatorname{arcsec}^{-2}$

where $N(\Delta m \leq 3)$ is the total number of galaxies within 3 magnitudes of the brightest member, $\mu$ is the effective surface brightness averaged over the smallest enclosing circle with angular radius $\theta_{G}, \theta_{n}(\Delta m \leq 3)$ is the angular radius of the largest concentric circle that contains no external galaxies within the same magnitude range. With follow-up spectroscopic measurements, Hickson et al. (1992) further removed 8 HCGs which contain fewer than three accordant members. This formed a tradition of the CG selection: searching the CG candidates that satisfy all the Hickson's criteria in the first step, then discarding the groups containing interlopers according to their redshifts. Thereafter, many CG catalogs have been constructed following this methodology: from the COSMOS-UKST Southern Galaxy Catalog (Iovino 2002); from the DPOSS Catalog (Iovino et 
al. 2003); from the Two Micron All Sky Survey (2MASS) extended source catalog (Díaz-Giménez et al. 2012) and from the Sloan Digital Sky (SDSS) galaxy catalog (Lee et al. 2004, McConnachie et al. 2009, Sohn et al. 2015). This kind of post-HCG procedure is very efficient in identifying real CGs, which however inherits a bias against nearby CGs in its photometric selection. For the nearby CGs, because of its large angular diameter,the isolation criteria is more likely to be broken given the background galaxies are uniformly distributed on the sky. (See Fig. 1 for an illustration).

To overcome the above selection bias in photometric CG samples, Barton et al. (1996) introduced an alternative approach: the friend-of-friend (FoF) algorithm, which identifies whether two galaxies belong to the same group using their projected separation and line-of-sight (LOS) velocity difference. Several authors identified CGs following this method from various redshift surveys, including CfA2 (Barton et al. 1996) and 2dFGRS (Saucedo-Morales \& Loera-González 2007), and from the SDSS main galaxy sample (MGS) (Sohn et al. 2016). This FoF algorithm has a strong dependence on the redshift completeness of the sample galaxies. Modern galaxy redshift surveys typically use a multifiber spectrograph, where the fiber collision effect brings an incompleteness effect at small angular scale. For example, the fibers cannot be placed closer than 55" on the same plate in the SDSS (Zehavi et al. 2002), which leads to a very high spectroscopic incompleteness on the compact galaxy system (Patton \& Atfield 2008; Shen et al. 2016). Moreover, distant CGs tend to be smaller in angular sizes and therefore are more likely to be biased by this incompleteness effect.

Recently, Díaz-Giménez et al. (2018) combined the advantages of the above two selection algorithms and applied their new algorithm on the galaxy catalog of SDSSDR12 (Alam et al. 2015). Specifically, they first apply a redshift filter to remove the background galaxies. These galaxies without redshifts are considered as background at this step. They then select the CG candidates inside the redshift slices using the Hickson's criteria. Finally, they bring those galaxies without redshifts back and check whether they could be the interlopers of these spectroscopic CG candidates. These groups without interlopers are finally confirmed as 'noncontaminated' CGs and otherwise listed as 'potentially contaminated' CGs. With the redshift slice to remove the background galaxies in the beginning, this algorithm effectively avoids the selection bias caused by the low redshift CGs in the traditional photometric-only selection technique.

However, in the algorithm of Díaz-Giménez et al. (2018), all the CG samples are initially selected based on spectroscopic galaxy samples. A real CG with spectroscopic incompleteness will be missed in the final CG sample if its spectroscopic members cannot pass the Hickson's criteria. To overcome this, we propose a revised algorithm to select CGs, where we keep rather than remove these galaxies without redshift measurements during the initial CG selection (see Section 3 for detail). With this new algorithm, we tend to retain all CG candidates, which could be easily verified with future spectroscopic survey, e.g. the complementary galaxies in the LAMOST spectral survey (Su, \& Cui 2004; Cui, et al. 2012; Luo et al. 2012). Actually, the redshift complete- a).
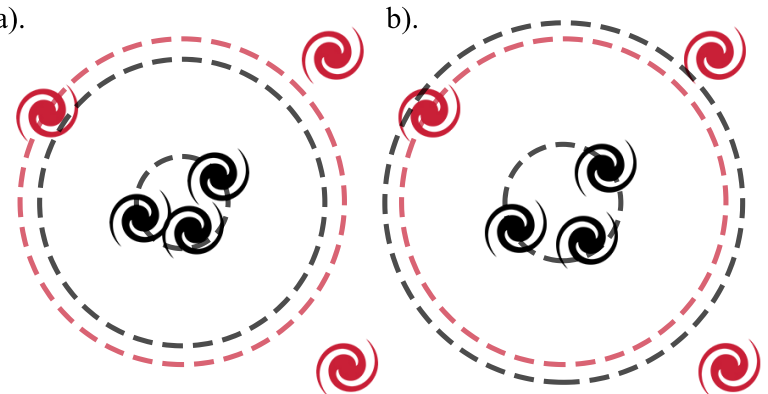

Galaxies with Similar Redshifts (C) Background Galaxies

FIG. 1.- Assumed compact group at different redshifts. The distant one (left panel) has smaller angular separation and is more likely to satisfy $\theta_{n} \geq 3 \theta_{G}$, while the nearby one (right panel) is more likely to violate the $\theta_{n} \geq 3 \theta_{G}$ criterion.

TABLE 1

Sources of Redshift Measurement

\begin{tabular}{lr}
\hline \hline \multicolumn{1}{c}{ Redshift Survey } & Redshift Number \\
\hline SDSS-DR14 & 694,930 \\
LAMOST (Till DR7-Q2) & 8,021 \\
GAMA-DR2 & 1,017 \\
Others from VAGC & 8,231 \\
Total Redshift Measurement & 712,199 \\
Total Galaxy Sample & 746,950 \\
\hline
\end{tabular}

ness of the SDSS main sample galaxies have already been significantly improved by the LAMOST spectral survey (Luo et al. 2015; Shen et al. 2016; Feng et al. 2019).

Therefore, the motivation of this study is to take the advantages of the new selection algorithm and the supplied redshifts from the LAMOST spectral survey to build the most complete and well-defined low- $z$ CG samples. In the next paper of this series, we will use this CG sample to study their dynamical properties and environmental dependence in detail. This paper is organized as follows. In section 2, we briefly describe the galaxy catalog used in this work. In section 3, we optimize the selection procedure for maximizing the CG samples and preserving all possible CG candidates. We describe the derived CG catalogs in section 3.4 and make comparisons with other available CG catalogs in section 4 . We provide a brief discussion on the final CG samples in section 5 and finally summarize our results in section 6 . Throughout this paper, we assume the flat WMAP7 cosmology with parameters: $H_{0}=70 \mathrm{~km} \mathrm{~s}^{-1} \mathrm{Mpc}^{-1}$ and $\Omega_{m}=0.27$ (Komatsu et al. 2011).

\section{GALAXY SAMPLES}

In this paper, we select the CGs in the largest and most complete low redshift spectroscopic galaxy sample, the MGS in the legacy of the SDSS, which is defined as the galaxies with $r$-band Galactic extinction corrected Petrosian magnitude $r \leq 17.77$ (Strauss et al. 2002). In SDSS, the bright galaxies are not complete in either photometric (deblending effect) or spectroscopic (saturation effect) sample (Strauss et al. 2002). Therefore, we also impose a bright-end limit $r \geq 14.00$ on our sample galaxies, following Lee et al. (2004). We take the basic photometric parameters and spectroscopic redshifts 
of the MGS from the NYU Value-Added Galaxy Catalog (Blanton et al. 2005, hereafter NYU-VAGC), which is based on DR7 of the SDSS (Abazajian et al. 2009).

Until the SDSS-DR7, there were about $\sim 7.0 \%$ of the MGS lacking spectroscopic redshifts due to the fiber collision effect. In NYU-VAGC, besides the spectroscopic redshifts from the SDSS DR7, extra redshifts are collected from 2dfGRs (Colless et al. 2001), 2MASS (Skrutskie et al. 2006), PSCz (Saunders et al. 2000) and RC3 (de Vaucouleurs et al. 1991). After DR7, the galaxies in the MGS without spectroscopy are continually targeted in the SDSS, which are also are targeted as a complementary galaxy sample in the LAMOST spectral survey (Luo et al. 2015). Following Feng et al. (2019), we matched the photometric MGS sample with the SDSSDR14, the most up-to-date LAMOST data release (DR7 V0, until March of 2019) and the GAMA-DR2 (Liske et al. 2016) and obtained a significant amount of extra redshifts. For these galaxies with more than one spectroscopy redshift, we set the priority as follow: SDSS $>$ LAMOST > GAMA > others from VAGC. Basically, the galaxy sample used in this study is an updated version of Feng et al. (2019) (updated with the newest data release of the LAMOST spectral survey), which contains 746,950 galaxies and has a spectroscopic completeness of $\sim 95.3 \%$. The detailed numbers of the global galaxy catalog and their spectroscopic redshifts from different surveys are listed in Table 1.

\section{COMPACT GROUPS}

\subsection{Selection Criteria}

In our CG sample selection, we slightly revise Hickson's criteria in the following way:

1. Richness: $3 \leq N(14.00 \leq r \leq 17.77) \leq 10$

2. Isolation: $\theta_{n} \geq 3 \theta_{G}$

3. Compactness: $\mu \leq 26.0 \mathrm{mag} \operatorname{arcsec}^{-2}$

4. Velocity Difference: $\left|V-V_{\text {med }}\right| \leq 1000 \mathrm{~km} \mathrm{~s}^{-1}$

where $V$ is the radial velocity of each member galaxy and $V_{\text {med }}$ is the median radial velocity of the members. Our new selection criteria are different from the traditional Hickson's one on two aspects. First, considering the fact that the triplet system is not distinguished from $N \geq 4$ groups (Duplancic et al. 2013), we extend the first criterion so as to include the triplets and maximize the sample size. We add an additional upper limit of $N \leq 10$ to distinguish the groups from rich clusters and accelerate the running time of the searching algorithm. Since the maximum richness of our derived groups contain $N=8$ member galaxies, this is not a very strict constraint. Second, we remove the constraint on the magnitude range $\Delta m \leq 3$, which is based on the consideration that the galaxies with similar magnitudes are more likely to be at similar redshifts. Here, our CG selection is based on spectroscopic galaxies, so the magnitude constraint is no longer necessary. Moreover, since our source galaxy catalog is in the magnitude range $14.00 \leq r \leq 17.77$, the resulting CGs will have $\Delta m_{r, \max }=3.77$ and therefore they are quite comparable to the traditional Hickson's CGs (see more discussion on this criteria in Section 5).

\subsection{Selection Procedure}

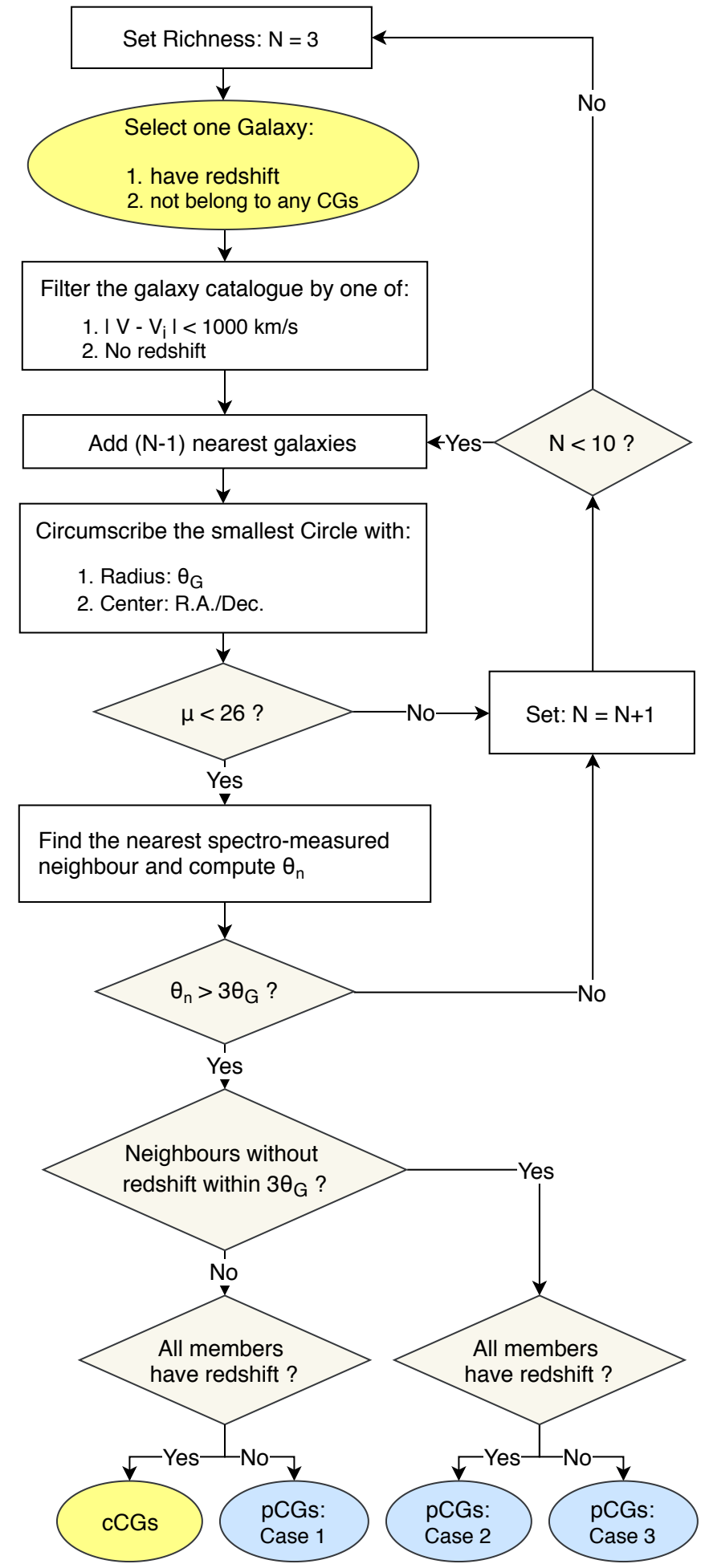

FIG. 2.- Flow chart of our CG selection procedure.

We show the flow chart of the selection procedure of the CGs and CG candidates in Fig.2. The main steps are outlined as follows:

Step 0: Start from a galaxy with spectroscopic redshift (recessional velocity $V_{i}$ ) in turn.

Step 1: Filter out the foreground and background galaxies and only keep galaxies with $\left|V-V_{i}\right|<1000 \mathrm{~km} / \mathrm{s}$. In this step, these galaxies in the same magnitude range 

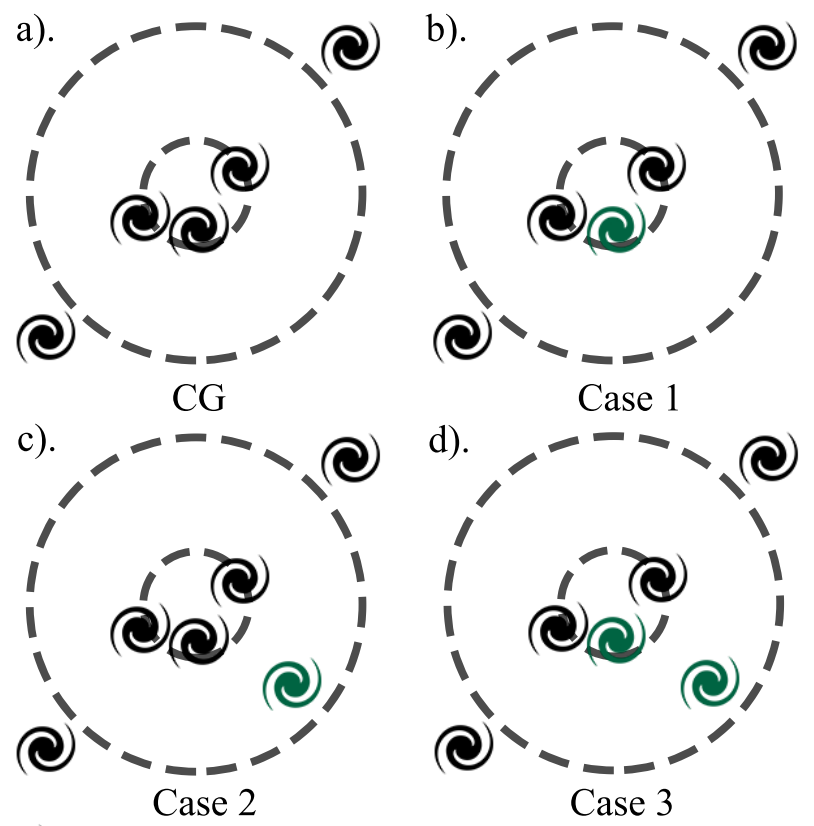

(C) Galaxies without Redshifts

Fig. 3.- Cartoons of the various cases of the CGs and CG candidates. (a) Conservative sample of compact groups: meet all requirement of CGs: meets all requirements of CG criteria. (b) Case 1: meets all CG criteria except that at least one member galaxy lacks spectroscopic redshifts. (c) Case 2: meets all CG criteria redshifts except that there are galaxies without spectroscopic redshift inside the isolation ring. (d) Case 3: Combination of Cases 1 and 2 .

TABLE 2

The total Numbers of THE CCGS AND PCGS AND THEIR MEMBER GALAXIES.

\begin{tabular}{lccr}
\hline \hline \multicolumn{2}{c}{ Catalog } & Groups & Member Galaxies \\
\hline \multirow{2}{*}{ cCGs } & $\ldots$ & 6,144 & 19,465 \\
\hline \multirow{2}{*}{ pCGs } & Case 1 & 6,790 & 21,382 \\
& Case 2 & 458 & 1,389 \\
& Case 3 & 774 & 2,361 \\
\cline { 2 - 4 } & Total & 8,022 & 25,132 \\
\hline
\end{tabular}

but without redshift measurements are considered as the same recessional velocity as $V_{i}$.

Step 2: Find the smallest circle that encircles $3 \leq N \leq$ 10 members. Write the radius of this circle as $\overline{\theta_{G}}$ and mark the center of this circle as the coordinate of the candidate CG. Calculate the mean surface brightness inside the circle and check the compactness criterion.

Step 3: Find the nearest neighbor galaxy with spectroscopic redshift, write the distance to the CG center as $\theta_{n}$. In this step, all the galaxies in the neighboring region without redshifts are considered as background galaxies. Check the isolation criteria. These CG candidates do not pass the isolation criteria, set $N=N+1$ and go back to Step 2.

Step 4: Separate CG candidates into 4 different catalogs according to the redshift status of galaxies. One is the conservative sample of CGs (hereafter cCGs) with spectroscopic redshifts for all the members and the nearest neighbor, the others with assumed redshifts are named as possibly CGs (hereafter pCGs). For the pCGs, there are three different kinds of cases.

1. Case 1: At least one of the members lacks redshift data but the others have genuine spectroscopic redshifts.

2. Case 2: All the members have genuine spectroscopic redshifts but at least one galaxy without redshift lies in the isolation rings.

3. Case 3: The combination of Cases 1 and 2.

The cartoon of the configurations of four types of CGs are shown in Fig. 3. In our selection procedure, we do not consider the extreme case, where one CG is possibly embedded in a larger $\mathrm{CG}$, i.e., our selection procedure stops once a CG or CG candidate is identified.

Step 5: Visual inspections on the images of all CGs. With this step, we remove some fake sources from bad photometry (e.g. McConnachie et al. 2009). Most of the contamination cases are caused by the effect that a large extended galaxy is split into many smaller parts and identified as isolated galaxies respectively.

With the above procedure, we finally obtain 6,144 cCGs and 8,022 pCGs. The detailed number of each type CGs and the number of their members are listed in Table 2.

For illustration, we show example SDSS images of each type $\mathrm{CG}$ and $\mathrm{CG}$ candidate in Appendix B.

\subsection{Velocity Dispersion of cCGs}

We estimate the rest-frame LOS velocity dispersion of our samples of CGs using the gapper estimator (Beers et al. 1990). Díaz-Giménez et al. (2012) suggests that the gapper estimator shows much fewer biases for groups with small numbers of members than the standard estimator of dispersion. For the ordered set of recession velocities $\left\{V_{i}\right\}$ of $N$ group members, the gaps are defined by:

$$
g_{i}=V_{i+1}-V_{i}, \quad i=1,2, \cdots, N,
$$

and the rest-frame velocity dispersion is estimated by

$$
\sigma_{\mathrm{LOS}}=\frac{\sqrt{\pi}}{\left(1+z_{g}\right) N(N-1)} \sum_{i=1}^{N-1} w_{i} g_{i}
$$

, where $z_{g}$ is the group redshift and $w_{i}$ is the Gaussian weight defined as $w_{i}=i(N-i)$.

\subsection{Compact Group Catalogs}

We present the final catalogs of 6,144 cCGs and 8,022 pCGs in Table 3 and 4, respectively.

In these two tables, we list their Group ID, sky coordinates (R.A. \& decl.), redshift, richness, angular radius, surface brightness and $\Delta m \leq 3$ criterion flag for each table. For each CG, the sky coordinate and angular radius are the center and radius of the minimum circle that encircles the group members. The surface brightness is thus the average surface brightness of the group members inside that circle. The redshift is the average redshifts of all group members, where the members in the pCGs with our redshift measurements have not been taken into account. The $\Delta m \leq 3$ criterion flag indicates whether all 
the group members are inside a magnitude range $\Delta m \leq 3$ ('0' for 'False'; ' 1 ' for 'True', see more discussion in section 5.1). In table 3, we list the LOS velocity dispersion of each CG, while in table 4 we provide the 'case flag' to show which groups belong to which case of pCGs as that demonstrated in Fig. 3.

Table 5 and table 6 list the properties of member galaxies of each cCGs and pCGs, respectively, including group ID, member ID, sky coordinates, redshift, Galactic extinction corrected $r$-band Petrosian magnitude, and spectroscopy data source. In table 6 , we also list the possible interlopers inside the isolation ring (i.e. $\left.\theta_{G}<\theta<3 \theta_{G}\right)$ of Case 2 and Case 3 pCGs, which have been assumed as background galaxies during the CG identification for completeness. These galaxies are assigned with member ID '- 99 ' for its corresponding groups. With this information, the Case 2 and Case 3 pCGs could be easily identified with future spectroscopic redshifts. For each catalog, only a few part of them are listed here. Full versions of these tables are available online in readable format.

We show the basic statistical properties of our cCG catalog in Fig.4, where the distributions of the richness $(N)$, redshift $\left(z_{G}\right)$, angular radius $\left(\theta_{G}\right)$ and velocity dispersion $\left(\sigma_{\mathrm{LOS}}\right)$ are plotted as the hatched histograms in the upper left, upper right, lower left, and lower right panel respectively. The richness of our cCGs spans a range from 3 to 8 and their redshift distribution peaks at $z \sim 0.08$. The typical angular radius of the cCGs is about $\sim 1^{\prime}$, indicating their compact nature. The distributions of these three apparent parameters are primarily resulted from the magnitude limit of our galaxy sample $(14.00 \leq r \leq 17.77)$ and our CG selection algorithm. We will present a detailed comparison of our cCGs with other CG catalogues that selected from the same SDSS galaxy sample on these distributions in Section 4. The velocity dispersion $\sigma_{\mathrm{LOS}}$, which characterizes the dynamical properties of the galaxy groups, is more robust against the selection effects. Our cCGs show similar $\sigma_{\text {LOS }}$ distribution as other catalogues in the range from 50 to 600 $\mathrm{km} / \mathrm{s}$, which is basically consistent with the expectation of normal galaxy groups (see more discussions in Section 5.1).

\section{COMPARISON WITH OTHER CG CATALOGS}

In this section, we compare our cCGs with other CG catalogs derived from the SDSS.

1. S15: McConnachie et al. (2009) derived the CG samples using Hickson criteria from the SDSS-DR6 photometric catalog (Adelman-McCarthy et al. 2008) in two ranges of magnitude limit and resulted in two data sets, Catalog A and B. Later, Sohn et al. (2015) supplied redshifts from FLOW/FAST observations and the SDSS-DR12, using the velocity filter to check the candidates of Catalog A. The final filtered sample (S15, hereafter) is comprised of 332 CGs with at least three member galaxies. This catalog is an implementation of the traditional searching procedure of CGs.

2. MLCG: Sohn et al. (2016) applied an FoF algorithm on the enhanced SDSS-DR12 magnitudelimited redshift survey and extracted a catalog of 1,588 CGs (MLCG, hereafter). Their FoF algo- rithm found the neighboring galaxies within a fixed projected distance and velocity difference. They also applied the similar richness and compactness criteria as used by McConnachie et al. (2008), but the isolation criterion was not taken into account.

3. HMCG: As we already mentioned, Díaz-Giménez et al. (2018) applied a redshift filter on galaxy sample before starting the Hickson's criteria to search CGs, where a sample of 462 CGs are finally extracted from the SDSS-DR12.

We summarize the methodology of the selection algorithm and the main features of the above CG catalogs in table 7. We show the distributions of the richness, redshift, angular radius, and LOS velocity dispersion of the CGs in these catalogs in Fig. 4. Next, we discuss and compare them with our cCGs one by one.

\section{1. cCGs versus $S 15$}

The identification of S15 is consistent with the implementation of the traditional Hickson's selection procedure. As expected, the relative fraction of nearby groups is lower than cCGs as shown in the upper right panel of Fig.4.

To make a detailed comparison with $\mathrm{S} 15$, we remove 52 CGs from S15 sample, which have at least one member galaxy without spectroscopic redshifts and would be identified as Case 1 pCGs in our study. We match the remaining 280 S15 CGs with our cCGs based on the angular separation and radial velocity difference and find 220 out of $280(\sim 78.6 \%)$ overlaps. The mismatched groups are mainly attributed to the small differences in the selection criteria, where S15 draws the galaxy catalog with $14.50 \leq r \leq 18.00$ and takes the restrictive condition $\Delta m \leq \overline{3}$ of the standard Hickson's criteria (see more discussions in Section 5).

\section{2. cCGs versus $M L C G s$}

For MLCGs, as we have introduced, the FoF selection algorithm biases against the high redshift objects in the SDSS. The projected distance restriction used in MLCGs is $D_{\text {lim }}=50 h^{-1} \mathrm{kpc}$, which corresponds to $55^{\prime}$ at redshift $z \sim 0.07$. Also, the exclusion of the isolation criterion and the inclusion of very bright galaxies even make the redshift distribution of the MLCGs be biased toward low redshifts as the upper right panel of Fig. 4 shows.

We also match the MLCGs with our cCGs and find that 736 out of $1,588(\sim 46.3 \%)$ MLCGs overlap with cCGs. This low match rate is mainly attribute to the neglect of isolation criterion in MLCGs. As mentioned in Sohn et al. (2016), 1,228 ( 77.0\%) MLCG systems violated Hickson's original isolation criterion. We find that 352 MLCGs violate our modified isolation criterion. Another cause of the difference is the inclusion of the bright $(r \leq 14.00)$ galaxies in MLCGs, which have been excluded in our study. There are $239(\sim 15.1 \%)$ MLCGs that contain these very bright members, most of them are located very nearby $(z \leq 0.05)$. That is to say, MLCGs are a very good complementary sample to our CGs, especially at low redshifts.

\section{3. cCGs versus HMCGs}

HMCGs comprises 406 noncontaminated CGs and 56 potentially contaminated CGs. Although our study 
TABLE 3

Catalog of the CCGs, which includes the Columns of Group ID, Sky Coordinates, Richness, Redshift, angular Radius, SURFACE BRIGHTNESS, LOS VELOCITY DISPERSION, AND $\Delta m \leq 3$ CRITERION FlaG ('0' FOR 'FALSE'; ' 1 ' FOR 'TRUE').

\begin{tabular}{lccccccc}
\hline \hline Group ID & $\begin{array}{c}\text { R.A. } \\
(\mathrm{J} 2000)\end{array}$ & $\begin{array}{c}\text { Decl. } \\
(\mathrm{J} 2000)\end{array}$ & $\mathrm{N}$ & $z_{g}$ & $\begin{array}{c}\theta_{G} \\
(\operatorname{arcmin})\end{array}$ & $\begin{array}{c}\mu \\
\left(\mathrm{mag} / \operatorname{arcsec}^{2}\right)\end{array}$ & $\begin{array}{c}\sigma_{\mathrm{LOS}} \\
(\mathrm{km} / \mathrm{s})\end{array}$ \\
\hline cCGs-0001 & 56.1382 & 1.0558 & 3 & 0.10425 & 0.526 & 24.265 & 484.706 \\
cCGs-0002 & 57.2751 & 0.8998 & 3 & 0.10925 & 0.993 & 25.965 & 68.366 \\
cCGs-0003 & 239.8562 & -0.9450 & 3 & 0.10238 & 0.463 & 24.133 & 340.199 \\
cCGs-0004 & 169.7778 & -0.2108 & 3 & 0.09746 & 0.466 & 24.185 & 398.755 \\
cCGs-0005 & 241.2247 & -0.0601 & 3 & 0.05093 & 1.328 & 25.784 & 287.055 \\
cCGs-0006 & 246.1077 & 0.8071 & 3 & 0.05834 & 1.160 & 25.681 & 503.839 \\
cCGs-0007 & 174.9150 & -1.0894 & 3 & 0.07777 & 0.622 & 24.721 & 412.498 \\
cCGs-0008 & 176.5369 & -1.1010 & 5 & 0.11765 & 1.200 & 25.475 & 390.835 \\
cCGs-0009 & 177.1710 & -1.1997 & 3 & 0.10660 & 0.312 & 23.896 & 415.169 \\
cCGs-0010 & 177.2054 & -1.1776 & 3 & 0.10647 & 0.488 & 24.590 & 1 \\
\hline
\end{tabular}

Note. This table has 6144 rows, of which only the first 10 rows are displayed here.

TABLE 4

Catalog of the pCGs, which includes the Columns of Group ID, Sky Coordinates, Richness, Redshift, angular Radius, SURFACE BRIGHTNESS, THE CLASSIFICATION OF UNCERTAIN CASE AS DESCRIBED IN FIG. 3 , AND $\Delta m \leq 3$ CRITERION FLAG.

\begin{tabular}{|c|c|c|c|c|c|c|c|c|}
\hline Group ID & $\begin{array}{l}\text { R.A. } \\
\text { (J2000) }\end{array}$ & $\begin{array}{l}\text { Decl. } \\
\text { (J2000) }\end{array}$ & $\mathrm{N}$ & $z_{g}$ & $\begin{array}{c}\theta_{G} \\
\text { (arcmin) }\end{array}$ & $\begin{array}{c}\mu \\
\left(\mathrm{mag} / \operatorname{arcsec}^{2}\right)\end{array}$ & Case & $\Delta m \leq 3$ \\
\hline pCGs-0001 & 241.3722 & -0.5285 & 3 & 0.13029 & 0.555 & 24.924 & 1 & 1 \\
\hline pCGs-0002 & 242.1523 & -0.1234 & 3 & 0.13994 & 0.888 & 25.946 & 2 & 1 \\
\hline pCGs-0003 & 242.6850 & -0.1302 & 3 & 0.10632 & 0.846 & 25.403 & 1 & 1 \\
\hline pCGs-0004 & 239.8296 & 0.3824 & 4 & 0.13990 & 1.322 & 25.898 & 1 & 1 \\
\hline pCGs-0005 & 239.8340 & 0.3660 & 4 & 0.09341 & 1.123 & 25.410 & 1 & 1 \\
\hline pCGs-0006 & 242.6550 & 0.3033 & 3 & 0.08022 & 0.568 & 24.665 & 3 & 1 \\
\hline pCGs-0007 & 242.6661 & 0.3009 & 3 & 0.05806 & 0.367 & 23.098 & 1 & 1 \\
\hline pCGs-0008 & 242.7184 & 0.3139 & 3 & 0.10622 & 0.736 & 24.834 & 1 & 1 \\
\hline pCGs-0009 & 242.5358 & 0.7680 & 3 & 0.04192 & 0.204 & 20.544 & 1 & 1 \\
\hline pCGs-0010 & 243.3732 & 0.8159 & 3 & 0.08196 & 0.419 & 24.480 & 1 & 1 \\
\hline
\end{tabular}

Note. This table has 8022 rows, where only the first 10 rows are displayed.

shares similar selection algorithm and galaxy catalog as those of HMCGs, the differences between two samples are significant. HMCGs are selected with a strict magnitude limit criterion that the brightest CG member should be at least three magnitudes brighter than the completeness magnitude of the galaxy sample $\left(r_{b} \leq r_{\lim }-3\right)$, which ensures the homogeneity of their CGs at different redshift (see further discussion in Section 5.2). Considering $r_{\text {lim }} \sim 17.77$ for the SDSS MGS, the brightest galaxy members of all HMCGs are therefore brighter than 14.77. Moreover, during the selection of HMGCs, there is no bright magnitude limit on the sample galaxies, while our CGs are selected on galaxies with $r \geq 14.00$. Combining these two effects, the redshift distribution of HMCGs is significantly biased to lower redshifts as the upper right panel of Fig.4 shows. The third difference of the HMCG selection from our study is that they adopt a fainter brightness criterion down to $\mu \leq 26.33$ mag $\operatorname{arcsec}^{-2}$. Combining the low redshift selection bias and the lower surface brightness criterion, HMCGs have significantly larger angular diameters than all other CG catalogs (lower left panel of Fig.4).

To make a fair comparison, we remove the HMCGs that either contain very bright members $(r<14.00)$ or with the surface brightness $\mu \geq 26.00 \mathrm{mag} \mathrm{arcsec}^{-2}$ and 218 groups are remain. We cross-match these 218 HMCGs with our cCGs and find 121 of them are overlapped. The other HMCGs that not listed in our cCGs are mainly caused by the wider magnitude range of the sample galaxies $(14.00 \leq r \leq 17.77)$ used in our CG selection than in HMCGs $\left(r_{b} \leq r \leq r_{b}+3\right)$.

These additional galaxies $\left(r_{b}+3 \leq r \leq 17.77\right)$, depending on their redshifts, might change the result of $\mathrm{CG}$ selection. If these additional galaxies were background galaxies, that would not change the CG identification. On the other hand, if these additional galaxies had accordance redshifts with the corresponding CGs, then they would be absorbed as the CG members and might not pass the isolation criterion in the next step of our CG selection. We show an example of such a case in Figure 9 of the Appendix B for illustration.

The differences among the final CG samples, resulted from the subtle differences of the selection criteria, indicates that the selection of a unique $\mathrm{CG}$ sample is quite nontrivial.

\section{DISCUSSION \\ 5.1. Selection Criteria}

As we have shown in the bottom right panel of Fig. 4, the peak of the $\sigma_{\text {LOS }}$ distribution of our cCGs is smaller than $200 \mathrm{~km} \mathrm{~s}^{-1}$. In our CG selection, we use a velocity difference criteria $\Delta V=\left|V-V_{\text {med }}\right|$ smaller than $1000 \mathrm{~km}$ $\mathrm{s}^{-1}$. In general, this radial velocity difference restriction minimizes the interlopers with discordant redshifts while recovering systems similar to the original Hickson CGs (Woods et al. 2010). However, this large critical $\Delta V$ value brings doubt that it might be too large for low mass groups (e.g. these with $\sigma_{\text {LOS }}<200 \mathrm{~km} \mathrm{~s}^{-1}$ ). To test this effect, for each $\mathrm{cCG}$, we compare $\Delta V$ of each group member with its $\sigma_{\text {LOS }}$ and find most of our groups 

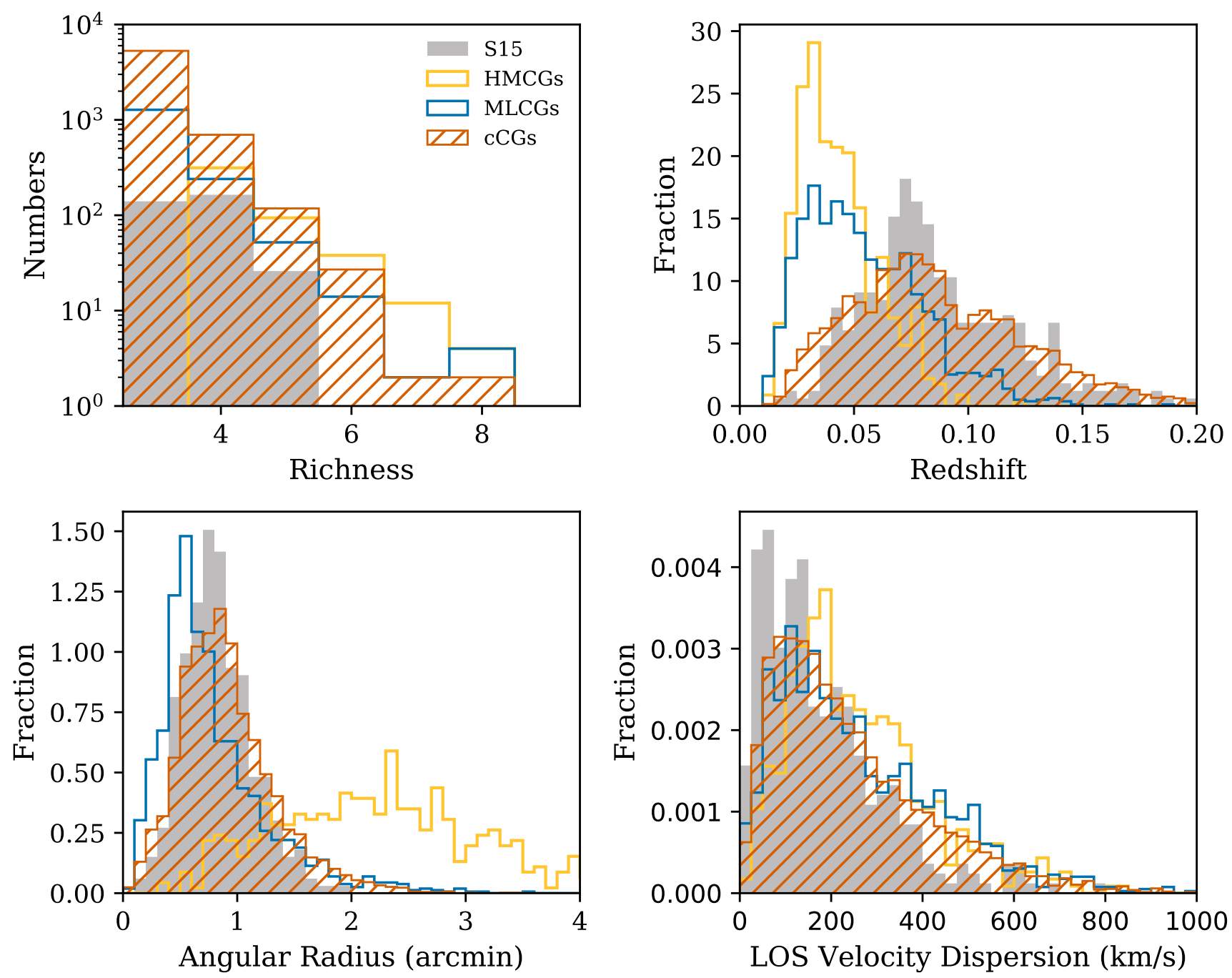

FIG. 4.- Comparison of the distributions of the CGs in various catalogs: cCGs (red hatched), HMCG (blue), MLCG (yellow), and S15-A (gray shaded). Upper left: group richness. Upper right: group redshift. Lower left: angular radius of the smallest enclosed circle. Lower right: velocity dispersion of the group.

have members with $\Delta V<2 \sigma_{L O S}$ and none of them with $\Delta V>3 \sigma_{\text {LOS }}$. This result may not be surprising. On the one hand, for CGs with few members, any group member with a large $\Delta V$ value would also bias $\sigma_{L O S}$ to a large value. On the other hand, as we will show in the next section, $\sigma_{\mathrm{LOS}}$ is nicely correlated with the total luminosity of the group members, which indicates that $\sigma_{\mathrm{LOS}}$ is a good dynamics indicator and therefore our cCGs could not be significantly contaminated by possible interlopers.

Nevertheless, we emphasize the critical $\Delta V$ value 1000 $\mathrm{km} \mathrm{s}^{-1}$ we adopt is somewhat arbitrary. A minor revision of this critical value will also slightly change our final CG catalog. For example, if we apply a tighter critical value, $\Delta V<800 \mathrm{~km} \mathrm{~s}^{-1}$, about $\sim 5 \%$ groups would be removed from current cCG sample. The critical value $1000 \mathrm{~km} \mathrm{~s}^{-1}$ we take is for the consistency with other studies, and also makes the comparison in Section 4 easier.

Also, during the construction of the CG samples, we do not restrict the group members within a magnitude range $\Delta m \leq 3$ as that typically used in other studies (e.g. McConnachie et al. 2009). As we mentioned in Section 3.1, the traditional Hickson criterion $\Delta m \leq 3$ is applicable to photometric-only galaxy samples, where the galaxies with similar magnitudes are more likely to have accordant redshifts. While our study is based on spectroscopic galaxy sample, we therefore do not need $\Delta m \leq 3$ to ensure the group nature of the selected galaxies. In our study, all CGs are selected in the magnitude range $14.00 \leq r \leq 17.77$ so that all of them have $\Delta m \leq 3.77$ and are comparable to the $\Delta m \leq 3$ that used in other studies (e.g. S15, HMCGs). Also, most of our CGs are located at $z>0.03$ and their brightest members have $r_{b} \geq 14.77$, which in turn makes most of the cCGs also satisfy $\Delta m \leq 3$. In fact, only $196 \mathrm{cCGs}(\sim 3.2 \%)$ and 297 pCGs $(\sim 3.7 \%)$ violate the $\Delta m \leq 3$ criterion. Apparently, these groups with $\Delta m \geq 3$ might be different from the traditional CGs. We therefore add a flag in the last column of Table 3 and 4 to call attention. We keep these groups with $\Delta m>3$ in our CG catalog based 

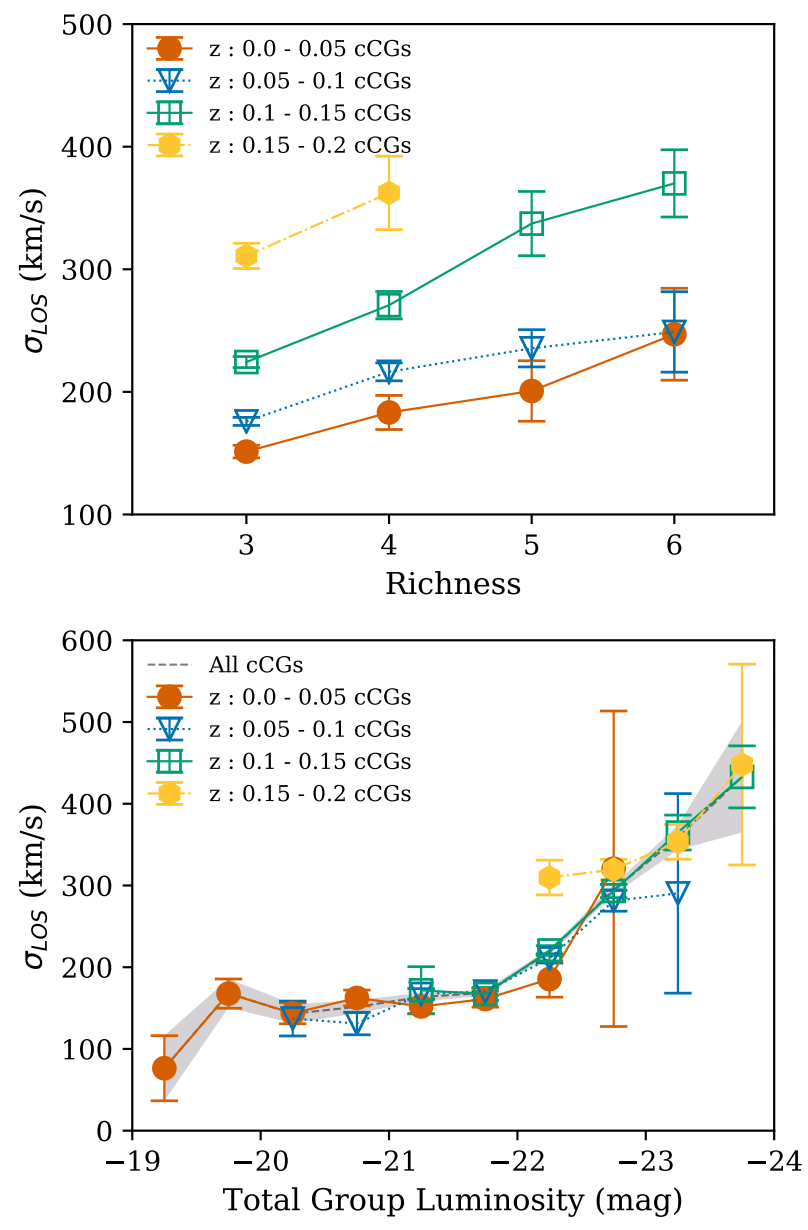

FIG. 5.- Median LOS velocity dispersion $\left(\sigma_{\mathrm{LOS}}\right)$ and its uncertainty as a function of group richness (upper panel) and total luminosity of observed members (lower panel) for cCGs at different redshift bins. Different symbols represent the CGs in different redshift bins as labeled in the top left corner of the figure. In the lower panel, the gray shaded area represents the same relation of all cCGs.

on two considerations. First, our CGs are selected from a magnitude-limited sample and the keeping of all the members inside the magnitude range $14.00 \leq r \leq 17.77$ makes the correction of selection effect (our next study) easier. Second, the compact nature of the CGs is ensured by the mean surface brightness of the group system (the compactness criterion), which is weakly affected by the inclusion of the faint galaxies. That is to say, the increasing of $\Delta m \leq 3$ to $\Delta m \leq 3.77$ has little impact on the compact nature of the selected groups.

Finally, we emphasize, for the magnitude-limited galaxy sample, a simple $\Delta m$ criterion (no matter its specific value) brings a significant inhomogeneity effect for groups at different redshifts. We make a detailed discussion next.

\subsection{Inhomogeneity of cCGs}

Our CGs are derived from a magnitude-limited sample of galaxies by applying a modified algorithm of the traditional Hickson Criteria, which brings a redshift dependent bias that makes the magnitude range of the group members be a function of its redshift. In fact, such a bias exists in all the CG samples based on magnitude-limited galaxy sample (e.g., S15, MLCG) except HMCG, where the brightest group galaxies are further required to fulfill $r_{b} \leq r_{\text {lim }}-3$. However, such a strong restriction in HMCG also makes the sample size small. Our study aims to maximize the CG sample size yet with 'well-defined' selection criteria and leave the correction of the sample selection effect in the upcoming study. Related to this, as we have discussed in Section 3.1, the relaxing of the traditional $\Delta m<3$ criterion to $14.00 \leq r \leq 17.77$ is also motivated by this consideration.

The inhomogeneity effect also makes the richness being a biased indicator of the group mass at different redshifts, as the upper panel of figure 5 shows; the median LOS velocity dispersion of cCGs $\left(\sigma_{\mathrm{LOS}}\right)$ is plotted as a function of their richness in different redshift bins. As can be seen, at a given richness, the median LOS velocity dispersion is systematically higher at higher redshifts.

To alleviate this inhomogeneity effect, it is better to use the total group luminosity rather than the richness to characterize the global group mass. A detailed calculation of the total luminosity of each $\mathrm{CG}$ requires the information of the CG members that have not been observed. Alternatively, the total luminosity could be estimated and corrected from the total luminosity of the observed members based on the conditional luminosity of group members (e.g., Yang et al. 2007, which will be preformed in the next work of our studies on the CGs. Nevertheless, we emphasize that the total luminosity of the observed members only is already a good proxy of the total luminosity of the CGs since the undetected CG members contribute a small fraction of the total luminosity of the CGs. To confirm this conclusion, we show the total luminosity of the observed CG members as a function of the LOS velocity dispersion for the cCGs in different redshift bins in the bottom panel of figure 5 . In contrast to the richness, these scaling relations show few biases in different redshift bins.

On the other hand, the missing of the bright galaxies $(r<14.00)$ in our selection criteria might introduce bias in the total luminosity of the group members. To validate this effect, we search the galaxies with $r<14.00$ within $3 \theta_{G}$ for each cCG. We find $76 r<14.00$ galaxies associating with 74 cCGs. Although our CGs are strictly defined on the galaxy sample with $14.00 \leq r \leq 17.77$, the group members and the total luminosity of these CGs with very bright association should be used with caution. To make compensate, we list these $r<14.00$ bright galaxies in appendix A. As listed, most of them are located at $z<0.03$.

Another possible inhomogeneity of our CG sample is the spectroscopic redshift source. As we have introduced in Section 2, the spectroscopic redshifts of our galaxy sample are heterogeneous. If there were systematical differences among different redshift catalogs, the LOS velocity dispersion measured for these CGs with heterogeneous redshifts would be biased to higher values. However, considering the typical uncertainty of the velocity measurements of current spectroscopy surveys $(\Delta V<10$ $\mathrm{km} \mathrm{s}^{-1}$ ), and we also have tested, this bias is negligible for our $\sigma_{\text {LOS }}$ measurements (see also Shen et al. 2016).

\section{3. $c C G$ s versus $p C G s$}

In this study, we have also obtained a sample of 8,022 pCGs that have not been discussed yet. We show the 
TABLE 5

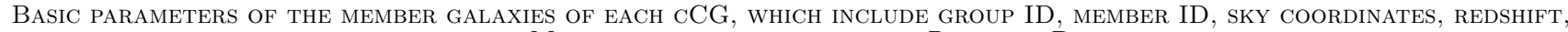
$r$-Band Magnitude And the Source of Redshift Data.

\begin{tabular}{|c|c|c|c|c|c|c|}
\hline Group ID & Member ID & $\begin{array}{c}\text { R.A. } \\
\text { (J2000) }\end{array}$ & $\begin{array}{c}\text { Decl. } \\
\text { (J2000) }\end{array}$ & $\mathrm{z}$ & rmag & Source ${ }^{*}$ \\
\hline cCGs-0001 & 1 & 56.1398 & 1.0471 & 0.10312 & 17.565 & 1 \\
\hline cCGs-0001 & 2 & 56.1366 & 1.0644 & 0.10349 & 16.929 & 1 \\
\hline cCGs-0001 & 3 & 56.1375 & 1.0544 & 0.10615 & 16.133 & 1 \\
\hline cCGs-0002 & 1 & 57.2892 & 0.8911 & 0.10910 & 16.755 & 1 \\
\hline cCGs-0002 & 2 & 57.2610 & 0.9085 & 0.10912 & 17.428 & 2 \\
\hline cCGs-0002 & 3 & 57.2689 & 0.8993 & 0.10953 & 17.040 & 1 \\
\hline cCGs-0003 & 1 & 239.8613 & -0.9393 & 0.10117 & 17.484 & 1 \\
\hline cCGs-0003 & 2 & 239.8510 & -0.9508 & 0.10268 & 16.262 & 1 \\
\hline cCGs-0003 & 3 & 239.8504 & -0.9445 & 0.10328 & 17.268 & 1 \\
\hline cCGs-0004 & 1 & 169.7703 & -0.2127 & 0.09620 & 16.237 & 4 \\
\hline cCGs-0004 & 2 & 169.7850 & -0.2138 & 0.09750 & 17.506 & 1 \\
\hline cCGs-0004 & 3 & 169.7718 & -0.2059 & 0.09867 & 17.498 & 1 \\
\hline cCGs-0005 & 1 & 241.2199 & -0.0385 & 0.05027 & 16.202 & 1 \\
\hline cCGs-0005 & 2 & 241.2295 & -0.0818 & 0.05055 & 16.848 & 1 \\
\hline cCGs-0005 & 3 & 241.2225 & -0.0475 & 0.05197 & 15.854 & 1 \\
\hline cCGs-0006 & 1 & 246.1209 & 0.7930 & 0.05699 & 17.100 & 1 \\
\hline cCGs-0006 & 2 & 246.0963 & 0.7914 & 0.05803 & 16.513 & 1 \\
\hline cCGs-0006 & 3 & 246.0957 & 0.8223 & 0.06000 & 15.940 & 1 \\
\hline cCGs-0007 & 1 & 174.9234 & -1.0833 & 0.07654 & 16.472 & 3 \\
\hline cCGs-0007 & 2 & 174.9079 & -1.0969 & 0.07772 & 17.034 & 3 \\
\hline cCGs-0007 & 3 & 174.9048 & -1.0872 & 0.07905 & 17.025 & 3 \\
\hline cCGs-0008 & 1 & 176.5291 & -1.0937 & 0.11589 & 16.259 & 3 \\
\hline cCGs-0008 & 2 & 176.5184 & -1.1038 & 0.11633 & 17.509 & 1 \\
\hline cCGs-0008 & 3 & 176.5396 & -1.0812 & 0.11855 & 16.642 & 1 \\
\hline cCGs-0008 & 4 & 176.5341 & -1.1208 & 0.11861 & 16.540 & 3 \\
\hline cCGs-0008 & 5 & 176.5346 & -1.1194 & 0.11886 & 16.890 & 1 \\
\hline cCGs-0009 & 1 & 177.1664 & -1.2022 & 0.10535 & 17.357 & 1 \\
\hline cCGs-0009 & 2 & 177.1699 & -1.1946 & 0.10649 & 17.670 & 3 \\
\hline cCGs-0009 & 3 & 177.1756 & -1.2021 & 0.10794 & 17.466 & 1 \\
\hline cCGs-0010 & 1 & 177.2069 & -1.1696 & 0.10577 & 17.285 & 1 \\
\hline cCGs-0010 & 2 & 177.2084 & -1.1829 & 0.10682 & 16.856 & 3 \\
\hline cCGs-0010 & 3 & 177.2039 & -1.1856 & 0.10682 & 17.615 & 1 \\
\hline
\end{tabular}

Note. The group information is listed in table 3. This table has 19,465 rows, only the first 32 rows are shown in here (corresponding to the first $10 \mathrm{cCGs}$ listed in table 3 ).

* Source of the galaxy redshift: '0' for no redshift measurement; '1' for SDSS; '2' for LAMOST; '3' for GAMA; '4' for VAGC others.

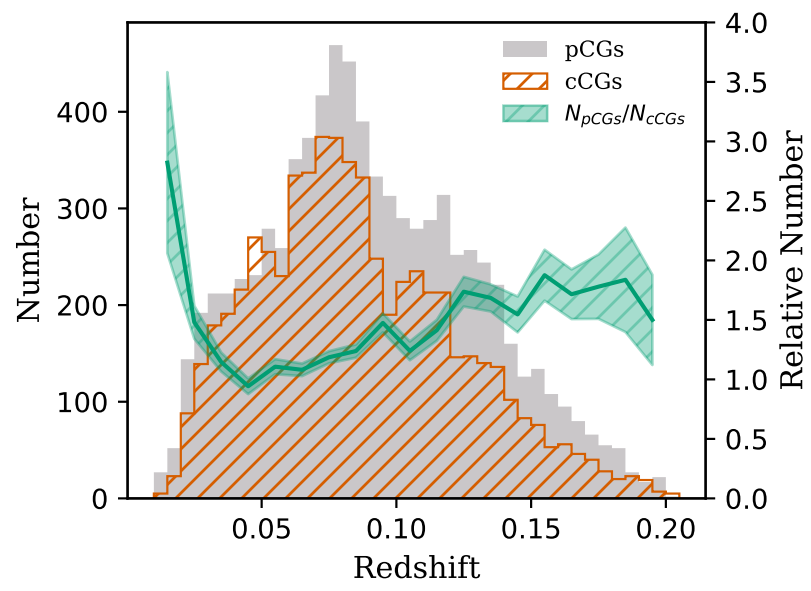

FIG. 6.- Redshift distributions of cCGs (red hatched) and pCGs (gray shaded). The green curve represents the ratio of the number of pCGs and cCGs at different redshift bins, the light region represents the $1 \sigma$ deviation from 1000 bootstraps.

redshift distributions of cCGs and pCGs as hatched and shaded histograms in Figure 6 respectively. For clarity, we show the number ratio of pCGs to cCGs as the green curve in Figure 6. This ratio shows a minimum at $z \sim$ 0.05 , and increases steeply and slowly toward the low and high redshift ends. This trend is to be expected: the

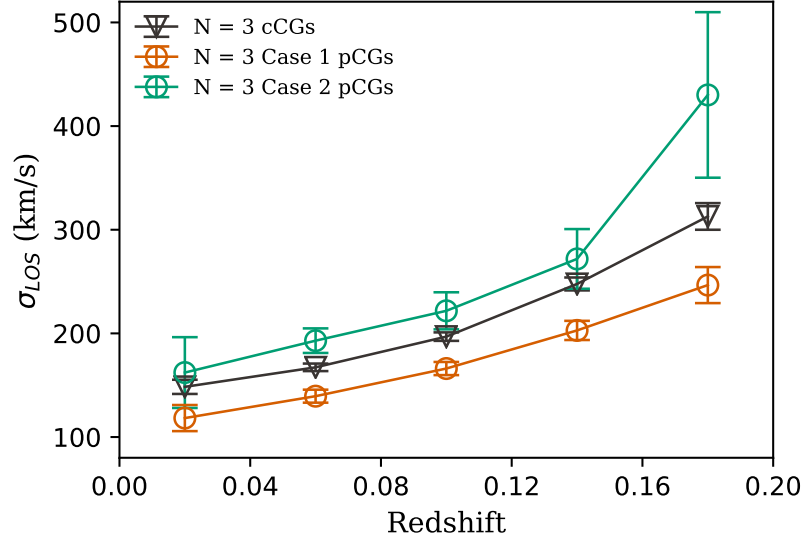

FIG. 7.- Median LOS velocity dispersion $\left(\sigma_{\mathrm{LOS}}\right)$ as a function of group redshift. The triangles, squares, and open circles represent $N=3 \mathrm{cCGs}, N=3$ Case $1 \mathrm{pCGs}$, and $N=3$ Case 2 pCGs respectively.

nearby pCGs have larger radius and are more likely to be contaminated by the background galaxies' chance to be lie within $3 \theta_{G}$; while for distant CGs, their angular radii are smaller on average, where the fiber collision effect becomes more significant and makes cCGs less complete.

We have also measured $\sigma_{\mathrm{LOS}}$ for pCGs, where the member galaxies without spectroscopic redshifts are sim- 
TABLE 6

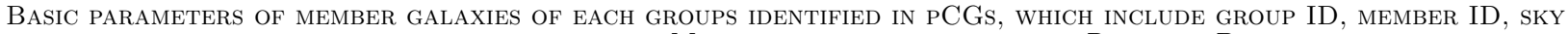
Coordinates, Redshift, $r$-Band Magnitude and the source of Redshift Data.

\begin{tabular}{|c|c|c|c|c|c|c|}
\hline Group ID & Member ID & $\begin{array}{l}\text { R.A. } \\
\text { (J2000) }\end{array}$ & $\begin{array}{c}\text { Decl. } \\
\text { (J2000) }\end{array}$ & $\mathrm{z}$ & rmag & Source \\
\hline pCGs-0001 & 1 & 241.3809 & -0.5255 & - & 17.569 & 0 \\
\hline pCGs-0001 & 2 & 241.3634 & -0.5315 & 0.12959 & 17.338 & 1 \\
\hline pCGs-0001 & 3 & 241.3788 & -0.5243 & 0.13099 & 16.963 & 1 \\
\hline pCGs-0002 & $-99^{*}$ & 242.1488 & -0.1411 & - & 17.764 & 0 \\
\hline pCGs-0002 & 1 & 242.1480 & -0.1375 & 0.13994 & 17.304 & 1 \\
\hline pCGs-0002 & 2 & 242.1566 & -0.1092 & 0.13991 & 17.518 & 1 \\
\hline pCGs-0002 & 3 & 242.1590 & -0.1264 & 0.14023 & 17.028 & 1 \\
\hline pCGs-0003 & 1 & 242.6924 & -0.1257 & - & 16.772 & 0 \\
\hline pCGs-0003 & 2 & 242.6894 & -0.1167 & 0.10626 & 17.149 & 1 \\
\hline pCGs-0003 & 3 & 242.6807 & -0.1436 & 0.10638 & 16.620 & 1 \\
\hline pCGs-0004 & 1 & 239.8467 & 0.3686 & - & 15.845 & 0 \\
\hline pCGs-0004 & 2 & 239.8125 & 0.3963 & 0.14048 & 17.345 & 1 \\
\hline pCGs-0004 & 3 & 239.8170 & 0.3658 & - & 16.776 & 0 \\
\hline pCGs-0004 & 4 & 239.8282 & 0.3653 & 0.13932 & 17.554 & 1 \\
\hline pCGs-0005 & 1 & 239.8170 & 0.3658 & - & 16.776 & 0 \\
\hline pCGs-0005 & 2 & 239.8523 & 0.3621 & 0.09352 & 16.894 & 1 \\
\hline pCGs-0005 & 3 & 239.8467 & 0.3686 & - & 15.845 & 0 \\
\hline pCGs-0005 & 4 & 239.8157 & 0.3699 & 0.09331 & 17.073 & 1 \\
\hline pCGs-0006 & $-99^{*}$ & 242.6693 & 0.3061 & - & 17.024 & 0 \\
\hline pCGs-0006 & 1 & 242.6529 & 0.3126 & 0.08022 & 16.965 & 1 \\
\hline pCGs-0006 & 2 & 242.6644 & 0.3029 & - & 16.778 & 0 \\
\hline pCGs-0006 & 3 & 242.6456 & 0.3017 & - & 17.138 & 0 \\
\hline pCGs-0007 & 1 & 242.6629 & 0.2957 & 0.05806 & 15.694 & 1 \\
\hline pCGs-0007 & 2 & 242.6644 & 0.3029 & - & 16.778 & 0 \\
\hline pCGs-0007 & 3 & 242.6693 & 0.3061 & - & 17.024 & 0 \\
\hline pCGs-0008 & 1 & 242.7062 & 0.3129 & 0.10622 & 15.816 & 1 \\
\hline pCGs-0008 & 2 & 242.7307 & 0.3150 & - & 17.304 & 0 \\
\hline pCGs-0008 & 3 & 242.7080 & 0.3187 & - & 17.274 & 0 \\
\hline pCGs-0009 & 1 & 242.5368 & 0.7697 & 0.04192 & 14.900 & 1 \\
\hline pCGs-0009 & 2 & 242.5338 & 0.7707 & - & 17.295 & 0 \\
\hline pCGs-0009 & 3 & 242.5378 & 0.7652 & - & 14.459 & 0 \\
\hline pCGs-0010 & 1 & 243.3677 & 0.8186 & 0.08188 & 17.370 & 1 \\
\hline pCGs-0010 & 2 & 243.3666 & 0.8184 & - & 17.344 & 0 \\
\hline pCGs-0010 & 3 & 243.3797 & 0.8135 & 0.08204 & 17.586 & 1 \\
\hline
\end{tabular}

Note. The corresponding group information is listed in table 4. This table has 26,652 rows, 25,132 rows correspond to the members of each pCGs and the rest 1,520 rows list the 'interlopers' of Case $2 \& 3$ pCGs (member ID: -99). Here we list the first 34 rows (corresponding to the first 10 pCGs listed in table 4).

* '-99' for the 'interlopers' of Case $2 \& 3$ pCGs.

ply masked. Since not all pCGs fulfill with the criteria of cCGs, we would expect that $\sigma_{\text {LOS }}$ of pCGS would be biased from that of cCGs. We show such a plot in Fig.7, where the average $\sigma_{\mathrm{LOS}}$ of $N=3$ cCGs, Case 1 pCGs and Case 2 pCGs are plotted as functions of redshift. As expected, because of the interloper effect, at a given redshift, Case 1 pCGs have lower $\sigma_{\text {LOS }}$ while Case 2 pCGs have larger $\sigma_{\mathrm{LOS}}$ than cCGs.

Ideally, if the redshift measurements of the SDSS MSGs are completed in the future, all our CG candidates, i.e. pCGs, could be redetermined as either real CGs (i.e. cCGs) or contaminators. Therefore, it is informative to have a estimation on the fraction of pCGs that could be identified as cCGs. To do that, we mask all the redshifts taken from the LAMOST spectral survey and make the same CG identification flow chart again. In this case, we obtain 1770 additional pCGs. Among them, 1010 are now identified as cCGs using LAMOST redshifts. Taking the fraction of pCGs that are cCGs (50\% - 60\%, see alsoMcConnachie et al. 2008), we estimate there are about $\sim 5000$ genuine CGs in our pCG catalog.

\section{SUMMARY}

In this paper, we present two catalogs of CGs identified from the SDSS main sample galaxies $(14.00 \leq r \leq 17.77)$ supplied with a significant fraction of redshifts from alternative surveys (e.g. LAMOST spectral survey and GAMA). Our motivation is to take the advantages of additional redshifts and maximize the final CG sample for statistical studies in next. Similar to Díaz-Giménez et al. (2018), our CG selection algorithm combines the advantages of two traditional $\mathrm{CG}$ selection algorithms, the photometric Hickson Criteria and spectroscopic FoF method, so as to avoid possible selection biases in either low or high redshifts. Our final genuine CG catalog (cCGs) contains 6,144 $N \geq 3$ groups with 19,465 member galaxies, and 8,022 CG candidates (pCGs) catalog with 25,132 members, which are the largest spectroscopic CG catalogs to date. We perform a detailed comparison of our CG catalog with other available CG catalogs (S15, MLCG, and HMCG). The difference and improvement of our CG selection algorithm are mainly reflected in the following way:

1. We extend the richness criterion to include the galaxy triplets.

2. We select the CGs in redshift slices, which prevents low- $z$ CGs being rejected if using photometric galaxy sample only.

3. We do not require $\Delta m \leq 3$ for CG members, which 
TABLE 7

Comparison between CCGs and other CG CATAlogues Derived From the SDSS.

\begin{tabular}{|c|c|c|c|c|c|}
\hline & & cCGs & S15 & MLCGs & HMCGs \\
\hline Galaxy & $\begin{array}{l}\text { SDSS Data Release } \\
\text { Magnitude System }\end{array}$ & $\begin{array}{l}\text { DR14+ } \\
\text { Petro }\end{array}$ & $\begin{array}{l}\text { DR12+ } \\
\text { Petro }\end{array}$ & $\begin{array}{c}\text { DR12+ } \\
\text { Model }\end{array}$ & $\begin{array}{c}\text { DR12+ } \\
\text { Model }\end{array}$ \\
\hline \multirow[t]{2}{*}{ Sample } & Bright End & 14.00 & 14.50 & - & - \\
\hline & Faint End & 17.77 & 18.00 & 17.77 & 17.77 \\
\hline \multirow{7}{*}{ Criteria } & Richness & $3 \leq N \leq 10$ & $N \geq 3$ & $N \geq 3$ & $4 \leq N \leq 10$ \\
\hline & Surface Brightness & $\bar{\mu} \leq 2 \overline{6.00}$ & $\mu \leq \overline{2} 6.00$ & $\mu \leq \overline{2} 6.00$ & $\bar{\mu} \leq 2 \overline{6.33}$ \\
\hline & Isolation & $\theta_{n} \geq 3 \theta_{G}$ & $\theta_{n} \geq 3 \theta_{G}$ & Unlimited & $\theta_{n} \geq 3 \theta_{G}$ \\
\hline & Projected Separation Limit & Unlimited & Unlimited & $\leq 50 h^{-1} \mathrm{kpc}$ & Unlimited \\
\hline & Radial Velocity Limit & $\leq 1000 \mathrm{~km} \mathrm{~s}^{-1}$ & $\leq 1000 \mathrm{~km} \mathrm{~s}^{-1}$ & $\leq 1000 \mathrm{~km} \mathrm{~s}^{-1}$ & $\leq 1000 \mathrm{~km} \mathrm{~s}^{-1}$ \\
\hline & Magnitude Limit & $14.00 \leq r \leq 17.77$ & $\Delta m \leq 3$ & $\Delta m \leq 3$ & $\begin{array}{c}\Delta m \leq 3 \\
r_{b} \leq 14.77\end{array}$ \\
\hline & Total Groups & 6,144 & 332 & 1,588 & 462 \\
\hline
\end{tabular}

significantly enlarges the sample size. The resulting inhomogeneity of the CG sample could be corrected in future statistical studies.

4. We keep all possible CG candidates in our pCG catalog, which could be identified with further new redshifts.

With this large cCG sample and new CGs replenished from the pCGs with future new redshifts (e.g. from LAMOST complementary galaxy sample, Shen et al. 2016), in our next work, we will perform a detailed statistical study on the physical nature of the CGs, e.g. dynamics, environment, and member galaxy properties etc.

This work is supported by National Key R\&D Program of China (No. 2019YFA0405501), National Natural Science Foundation of China (No.11573050 and 11433003) and Cultivation Project for LAMOST Scientific Payoff and Research Achievement of CAMS-CAS.

This work has made use of data products from the Sloan Digital Sky Survey (SDSS, http://www.sdss. org), the Large Sky Area Multi-Object Fiber Spectroscopic Telescope (LAMOST, http://www. lamost.org), the GAMA survey (http://www.gama-survey.org. We are thankful for their tremendous efforts on the surveying work.

Guoshoujing Telescope (the Large Sky Area MultiObject Fiber Spectroscopic Telescope LAMOST) is a
National Major Scientific Project built by the Chinese Academy of Sciences. Funding for the project has been provided by the National Development and Reform Commission. LAMOST is operated and managed by the National Astronomical Observatories, Chinese Academy of Sciences.

\section{APPENDIX}

\section{LIST OF THE BRIGHT GALAXIES WITHIN $3 \theta_{G}$ OF EACH CCGS}

In this appendix, we list the bright galaxies $(r<14.00)$ within $3 \theta_{G}$ of each cCGs in Table 8 , including sky coordinates, redshift, galactic extinction corrected $r$-band Petrosian magnitude, corresponding cCG ID, redshift of corresponding $\mathrm{cCG}$ and the projected location in corresponding cCG.

Since the bright galaxies in SDSS are easily contaminated by the deblending effect, we have made visual inspection for all these galaxies. There are 76 bright galaxies associated with $74 \mathrm{cCGs}$. If we join these bright galaxies into the current cCG catalog (defined on galaxies with $14.00 \leq r \leq 17.77), 26$ cCGs are still identified as cCGs (but the richness and surface brightness would be raised), while the other 48 would be rejected.

\section{EXAMPLE SDSS IMAGES OF CGS AND CG CANDIDATES}

Example SDSS images: Fig. 8 shows the images of different cases of cCGs and pCGs, Fig. 9 shows an example HMCG not identified as a cCG.

\section{REFERENCES}

Abazajian, K. N., Adelman-McCarthy, J. K., Agüeros, M. A., et al. 2009, ApJS, 182, 543-558

Adelman-McCarthy, J. K., Agüeros, M. A., Allam, S. S., et al. 2008, ApJS, 175, 297-313

Alam, S., Albareti, F. D., Allende Prieto, C., et al. 2015, ApJS, 219, 12

Barnes, J. E. 1989, Nature, 338, 123

Barton, E., Geller, M., Ramella, M., Marzke, R. O., \& da Costa, L. N. 1996, AJ, 112, 871

Beers, T. C., Flynn, K., \& Gebhardt, K. 1990, AJ, 100, 32

Blanton, M. R., Schlegel, D. J., Strauss, M. A., et al. 2005, AJ, 129, 2562

Brasseur, C. M., McConnachie, A. W., Ellison, S. L., \& Patton, D. R. 2009, MNRAS, 392, 1141

Coenda, V., Muriel, H., \& Martínez, H. J. 2012, A\&A, 543, A119

Colless, M., Dalton, G., Maddox, S., et al. 2001, MNRAS, 328, 1039

Coziol, R., \& Plauchu-Frayn, I. 2007, AJ, 133, 2630

Cui X.-Q., et al., 2012, RAA, 12, 1197 de Vaucouleurs, G., de Vaucouleurs, A., Corwin, H. G., Jr., et al. 1991, Third Reference Catalogue of Bright Galaxies. Volume I: Explanations and references. Volume II: Data for galaxies between $0^{h}$ and $12^{h}$. Volume III: Data for galaxies between $12^{h}$ and $24^{h}$., by de Vaucouleurs, G.; de Vaucouleurs, A.; Corwin, H. G., Jr.; Buta, R. J.; Paturel, G.; Fouqué, P.. Springer, New York, NY (USA), 1991, 2091

Deng, X.-F., He, J.-Z., \& Wu, P. 2008, A\&A, 484, 355

Diaferio, A., Geller, M. J., \& Ramella, M. 1994, AJ, 107, 868

Díaz-Giménez, E., Mamon, G. A., Pacheco, M., Mendes de Oliveira, C., \& Alonso, M. V. 2012, MNRAS, 426, 296

Díaz-Giménez, E., \& Mamon, G. A. 2010, MNRAS, 409, 1227

Díaz-Giménez, E., Zandivarez, A., \& Taverna, A. 2018, A\&A, 618, A157

Duplancic, F., O'Mill, A. L., Lambas, D. G., Sodré, L., \& Alonso, S. 2013, MNRAS, 433, 3547

Farhang, A., Khosroshahi, H. G., Mamon, G. A., Dariush, A. A., \& Raouf, M. 2017, ApJ, 840, 58

Feng, S., Shen, S.-Y., Yuan, F.-T., et al. 2019, ApJ, 880, 114 
TABLE 8

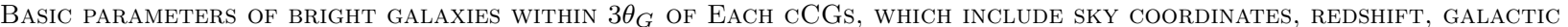
extinction Corrected $r$-BAnd Petrosian magnitude, Corresponding CCG ID, Redshift of Corresponding CCG and the Projected Location in Corresponding CCG

\begin{tabular}{|c|c|c|c|c|c|c|}
\hline $\begin{array}{l}\text { R.A. } \\
\text { (J2000) }\end{array}$ & $\begin{array}{c}\text { Decl. } \\
\text { (J2000) }\end{array}$ & $z$ & rmag & Group ID & $z_{G}$ & Location ${ }^{*}$ \\
\hline 31.3690 & 13.2516 & 0.02603 & 12.904 & cCGs-0226 & 0.02557 & 1 \\
\hline 24.7282 & 15.0216 & 0.02790 & 13.806 & cCGs-0236 & 0.02807 & 2 \\
\hline 131.4607 & 53.9925 & 0.03095 & 13.778 & cCGs-0462 & 0.03069 & 1 \\
\hline 121.3606 & 46.7078 & 0.02266 & 13.306 & cCGs-0503 & 0.02246 & 1 \\
\hline 212.8593 & 1.2865 & 0.02494 & 13.445 & cCGs-0571 & 0.02516 & 2 \\
\hline 216.1382 & 1.1773 & 0.03879 & 13.728 & cCGs-0573 & 0.03848 & 2 \\
\hline 230.0759 & 3.5183 & 0.03691 & 13.060 & cCGs-0730 & 0.03769 & 2 \\
\hline 27.2866 & 13.059 & 0.01743 & 13.963 & cCGs-0845 & 0.01725 & 1 \\
\hline 27.3085 & 13.0554 & 0.01693 & 12.488 & cCGs-0845 & 0.01725 & 1 \\
\hline 172.0738 & 2.6540 & 0.02282 & 13.799 & cCGs-0901 & 0.02263 & 1 \\
\hline 128.5946 & 48.0882 & 0.04294 & 13.906 & cCGs-0928 & 0.04341 & 1 \\
\hline 158.5600 & 61.6405 & 0.03114 & 13.576 & cCGs-0947 & 0.03061 & 2 \\
\hline 255.2513 & 39.5661 & 0.03426 & 13.719 & cCGs-1172 & 0.03417 & 1 \\
\hline 209.8532 & -3.2092 & 0.02448 & 13.399 & cCGs-1395 & 0.02475 & 1 \\
\hline 50.1789 & -1.1086 & 0.02091 & 13.890 & cCGs- 1670 & 0.02105 & 2 \\
\hline 50.1892 & -1.0447 & 0.02140 & 13.508 & cCGs-1670 & 0.02105 & 2 \\
\hline 177.7127 & 55.1437 & 0.0193 & 13.531 & cCGs-1714 & 0.01898 & 2 \\
\hline 139.1951 & 43.7126 & 0.0311 & 13.901 & cCGs-1894 & 0.02833 & 2 \\
\hline 187.1314 & 53.5961 & 0.03635 & 13.963 & cCGs-1917 & 0.03597 & 2 \\
\hline 199.1243 & 52.9337 & 0.0327 & 13.811 & cCGs-2189 & 0.03262 & 1 \\
\hline 173.9015 & 54.9486 & 0.01929 & 12.863 & cCGs-2225 & 0.01859 & 1 \\
\hline 45.9705 & 0.4152 & 0.04300 & 13.664 & cCGs-2364 & 0.04220 & 2 \\
\hline 155.6559 & 38.5791 & 0.05175 & 13.992 & cCGs-2626 & 0.05091 & 2 \\
\hline 145.8298 & 36.2478 & 0.02234 & 13.182 & cCGs-2636 & 0.02149 & 1 \\
\hline 228.3072 & 40.5379 & 0.03124 & 13.740 & cCGs-2658 & 0.03099 & 1 \\
\hline 218.2908 & 53.2328 & 0.04416 & 13.578 & cCGs-2836 & 0.04399 & 1 \\
\hline 187.5494 & 47.0063 & 0.03910 & 13.614 & cCGs- 2843 & 0.03989 & 2 \\
\hline 184.5536 & 44.1733 & 0.02453 & 13.664 & cCGs-2954 & 0.02474 & 1 \\
\hline 198.1661 & 12.5998 & 0.01121 & 12.876 & cCGs-3099 & 0.01135 & 1 \\
\hline 203.1773 & 7.3273 & 0.02338 & 13.030 & cCGs-3197 & 0.02372 & 2 \\
\hline 207.0208 & 7.3923 & 0.02328 & 13.903 & cCGs-3208 & 0.02313 & 2 \\
\hline 235.1470 & 28.3607 & 0.03271 & 13.806 & cCGs-3347 & 0.03063 & 1 \\
\hline 219.5425 & 9.3361 & 0.03029 & 13.678 & cCGs-3435 & 0.03086 & 2 \\
\hline 204.0058 & 6.5853 & 0.02195 & 13.634 & cCGs-3437 & 0.02222 & 2 \\
\hline 223.1732 & 7.9319 & 0.03552 & 13.664 & cCGs-3453 & 0.03546 & 2 \\
\hline 234.2215 & 4.7579 & 0.03909 & 13.97 & cCGs-3489 & 0.03907 & 2 \\
\hline 171.8910 & 36.0610 & 0.03462 & 13.862 & cCGs-4194 & 0.03448 & 2 \\
\hline 141.9700 & 29.9857 & 0.02666 & 13.130 & cCGs-4351 & 0.02627 & 2 \\
\hline 205.2964 & 30.3781 & 0.04039 & 13.888 & cCGs-4537 & 0.04019 & 1 \\
\hline 196.3090 & 31.9997 & 0.05188 & 13.912 & cCGs-4538 & 0.05183 & 2 \\
\hline 239.8022 & 20.7634 & 0.01466 & 13.605 & cCGs-4649 & 0.01416 & 2 \\
\hline 194.2975 & 29.0451 & 0.02496 & 13.749 & cCGs-4660 & 0.02512 & 2 \\
\hline 224.6263 & 23.9553 & - & 13.986 & cCGs-4673 & 0.04781 & 1 \\
\hline 241.5951 & 19.7780 & 0.03911 & 13.845 & cCGs-4802 & 0.03901 & 1 \\
\hline 219.0386 & 21.7935 & 0.01877 & 12.907 & cCGs-4866 & 0.01750 & 2 \\
\hline 236.2438 & 16.9621 & 0.04953 & 13.974 & cCGs-4869 & 0.04920 & 2 \\
\hline 226.7999 & 20.4796 & 0.04215 & 13.725 & cCGs-4878 & 0.04118 & 2 \\
\hline 167.6600 & 28.7676 & 0.03479 & 13.428 & cCGs-5058 & 0.03703 & 2 \\
\hline 184.9485 & 30.3391 & 0.02817 & 13.039 & cCGs-5084 & 0.02772 & 2 \\
\hline 139.2788 & 20.0697 & 0.02784 & 12.891 & cCGs-5116 & 0.02922 & 2 \\
\hline 195.4739 & 27.6244 & 0.02621 & 12.940 & cCGs-5166 & 0.02334 & 2 \\
\hline 181.5246 & 28.2380 & 0.02734 & 13.906 & cCGs-5171 & 0.02905 & 2 \\
\hline 168.0139 & 27.5898 & 0.03500 & 13.438 & cCGs-5230 & 0.03482 & 2 \\
\hline 188.9216 & 26.5231 & 0.02221 & 12.231 & cCGs-5232 & 0.02298 & 2 \\
\hline 194.1161 & 26.9874 & 0.02150 & 13.023 & cCGs-5239 & 0.02097 & 2 \\
\hline 194.8988 & 27.9593 & 0.02391 & 12.408 & cCGs-5248 & 0.02343 & 2 \\
\hline 164.6051 & 24.2263 & 0.02145 & 13.090 & cCGs-5312 & 0.02131 & 1 \\
\hline 162.1901 & 22.2178 & 0.04667 & 13.845 & cCGs-5320 & 0.04428 & 2 \\
\hline 170.6098 & 24.2991 & 0.02515 & 13.241 & cCGs-5330 & 0.02514 & 1 \\
\hline 144.7567 & 17.0253 & 0.02986 & 13.953 & cCGs-5371 & 0.02929 & 2 \\
\hline 174.4318 & 22.0098 & 0.03009 & 13.764 & cCGs-5389 & 0.03057 & 2 \\
\hline 213.2858 & 20.4163 & 0.01672 & 12.299 & cCGs-5414 & 0.01619 & 1 \\
\hline 120.5625 & 9.3944 & 0.01490 & 13.538 & cCGs-5457 & 0.01437 & 2 \\
\hline 167.4352 & 21.7589 & 0.03183 & 13.316 & cCGs-5492 & 0.03165 & 2 \\
\hline 175.6020 & 20.1193 & 0.01996 & 13.810 & cCGs-5593 & 0.02091 & 2 \\
\hline 176.1273 & 20.0767 & 0.02241 & 13.789 & cCGs-5594 & 0.02429 & 2 \\
\hline 181.0060 & 20.2323 & 0.02445 & 12.970 & cCGs-5595 & 0.02217 & 2 \\
\hline 181.0391 & 20.3479 & 0.02454 & 12.818 & cCGs-5596 & 0.02410 & 2 \\
\hline 174.6228 & 20.5277 & 0.02572 & 13.358 & cCGs-5603 & 0.02532 & 1 \\
\hline 227.0999 & 19.2081 & 0.02109 & 13.780 & cCGs-5613 & 0.02113 & 2 \\
\hline
\end{tabular}


TABLE 8

(Continued)

\begin{tabular}{ccccccc}
\hline \hline $\begin{array}{c}\text { R.A. } \\
(\mathrm{J} 2000)\end{array}$ & $\begin{array}{c}\text { Decl. } \\
(\mathrm{J} 2000)\end{array}$ & $z$ & rmag & Group ID & $z_{G}$ & Location $^{*}$ \\
\hline 243.2081 & 11.1598 & 0.04247 & 13.675 & cCGs-5653 & 0.04118 \\
199.3690 & 20.6123 & 0.02222 & 13.664 & cCGs-5666 & 0.02265 & 2 \\
213.0659 & 15.8419 & 0.01750 & 13.232 & cCGs-5786 & 0.01742 & 1 \\
221.8119 & 13.4564 & 0.02802 & 13.909 & cCGs-5806 & 0.02831 & 0.02513 \\
209.7617 & 15.5657 & 0.02559 & 13.135 & cCGs-5808 & 0.02913 \\
135.7972 & 13.6323 & 0.02903 & 13.554 & cCGs-5914 & 1 \\
\hline
\end{tabular}

Note. This table has 76 rows.

* Location of the bright galaxies in their corresponding cCGs: '1' for 'within the smallest enclosed circle'; '2' for 'within the isolation ring'
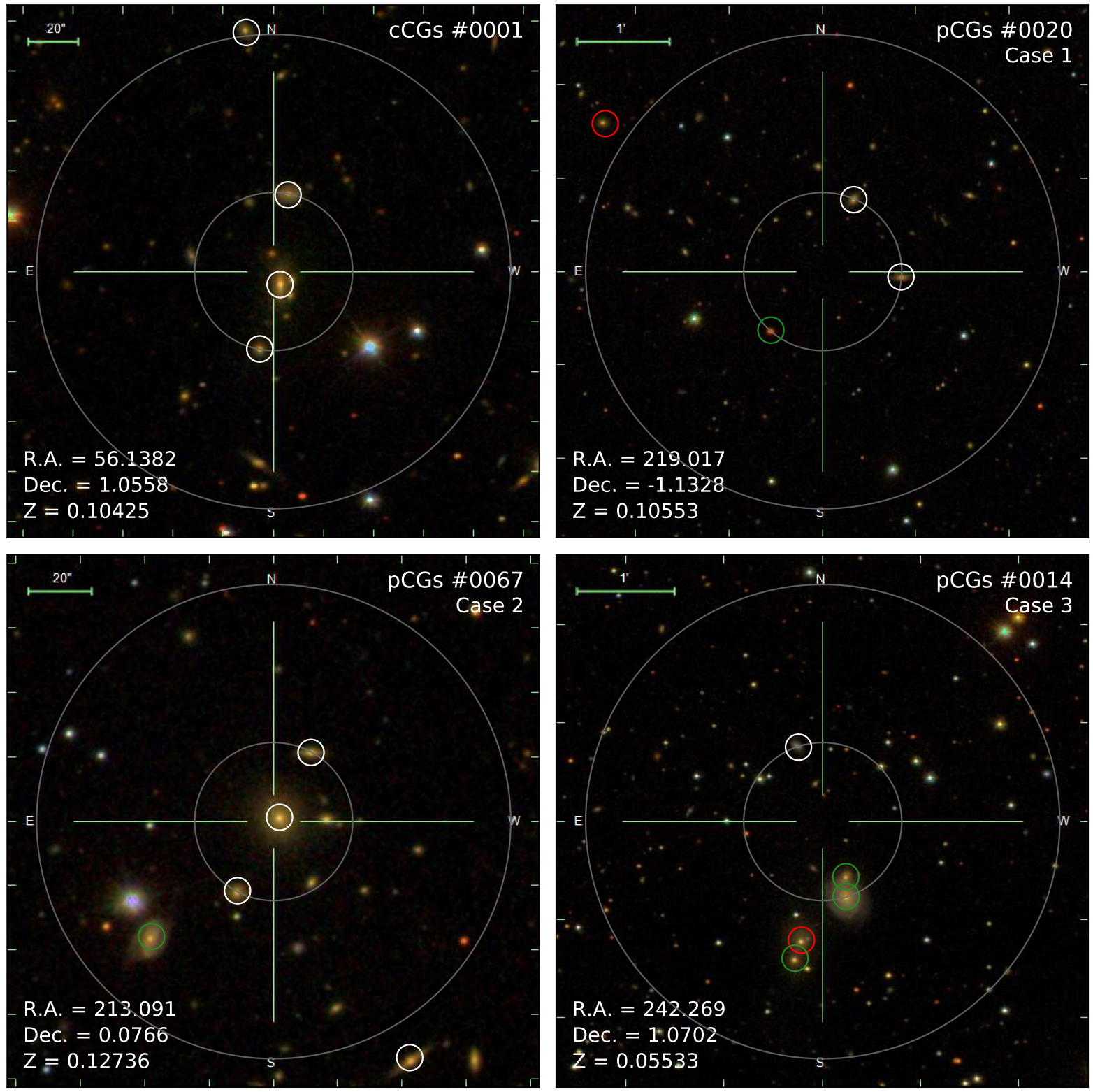

FIG. 8.- Example SDSS images of a cCG and each case of pCGs. For each CG image, the inner gray circle shows the smallest enclosed circles of the group members $\left(\theta_{G}\right)$, while outer gray circle represents the corresponding isolation ring $\left(\sim 3 \theta_{G}\right)$. The small white circles locate the group members with spectroscopic redshifts, while the small green circles label the galaxies without redshifts. The red circles represent the foreground or background galaxies. Only the galaxies in the magnitude range $14.00<r<17.77$ are labeled. 


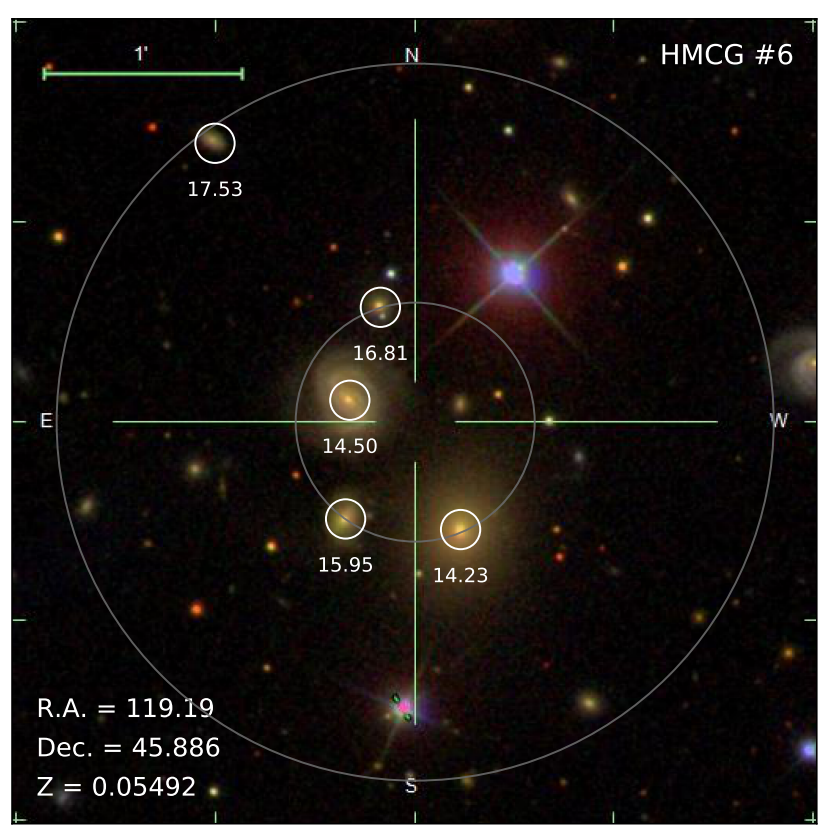

FIG. 9.- Example SDSS image of an HMCG that is not identified as a cCG. The symbol styles are the same as Fig.8, where the $r$ band magnitudes of each galaxies are also labelled. Because of the galaxy with $r=17.53$ located inside $3 \theta_{G}$, which is more than 3 magnitude fainter than the brightest member $(r=14.23)$ and has not been considered as a group member in HMCG, this group is not identified as a CG in our study.

Hickson, P. 1982, ApJ, 255, 382

Hickson, P., Mendes de Oliveira, C., Huchra, J. P., \& Palumbo, G. G. 1992, ApJ, 399, 353

Huchra, J. P., \& Geller, M. J. 1982, ApJ, 257, 423

Iovino, A. 2002, AJ, 124, 2471
Iovino, A., de Carvalho, R. R., Gal, R. R., et al. 2003, AJ, 125, 1660

Komatsu, E., Smith, K. M., Dunkley, J., et al. 2011, ApJS, 192, 18

Lee, B. C., Allam, S. S., Tucker, D. L., et al. 2004, AJ, 127, 1811 Liske, J., Baldry, I. K., Driver, S. P., et al. 2016, VizieR Online Data Catalog, 745

Luo, A.-L., Zhang, H.-T., Zhao, Y.-H., et al. 2012, Research in Astronomy and Astrophysics, 12, 1243

Luo, A.-L., Zhao, Y.-H., Zhao, G., et al. 2015, Research in Astronomy and Astrophysics, 15, 1095

McConnachie, A. W., Ellison, S. L., \& Patton, D. R. 2008, MNRAS, 387, 1281

McConnachie, A. W., Patton, D. R., Ellison, S. L., \& Simard, L. 2009, MNRAS, 395, 255

Moore, B., Lake, G., \& Katz, N. 1998, ApJ, 495, 139

Patton, D. R., \& Atfield, J. E. 2008, ApJ, 685, 235

Ponman, T. J., Allan, D. J., Jones, L. R., et al. 1994, Nature, 369,462

Saucedo-Morales, J., \& Loera-González, P. 2007, Groups of Galaxies in the Nearby Universe, 73

Saunders, W., Sutherland, W. J., Maddox, S. J., et al. 2000, MNRAS, 317,55

Shen, S.-Y., Argudo-Fernández, M., Chen, L., et al. 2016 , Research in Astronomy and Astrophysics, 16, 43

Skrutskie, M. F., Cutri, R. M., Stiening, R., et al. 2006, AJ, 131, 1163

Sohn, J., Hwang, H. S., Geller, M. J., et al. 2015, Journal of Korean Astronomical Society, 48, 381

Sohn, J., Geller, M. J., Hwang, H. S., Zahid, H. J., \& Lee, M. G. 2016, ApJS, 225, 23

Strauss, M. A., Weinberg, D. H., Lupton, R. H., et al. 2002, AJ, 124,1810

Su, D.-Q., \& Cui, X.-Q. 2004, Chinese J. Astron. Astrophys., 4, 1

Tempel, E., Tago, E., \& Liivamägi, L. J. 2012, A\&A, 540, A106

Woods, D. F., Geller, M. J., Kurtz, M. J., et al. 2010, AJ, 139, 1857

Yang, X., Mo, H. J., van den Bosch, F. C., et al. 2007, ApJ, 671, 153

Zehavi, I., Blanton, M. R., Frieman, J. A., et al. 2002, ApJ, 571, 172

Zhao, G., Zhao, Y.-H., Chu, Y.-Q., et al. 2012, Research in Astronomy and Astrophysics, 12, 723 